REGARDS

SUR L'ECONOMIE ALLEMANDE

BULLETIN ECONOMIQUE DU CIRAC
Regards sur l'économie allemande

Bulletin économique du CIRAC

$73 \mid 2005$

Varia

\title{
Années Schröder : les 'recentrages' successifs de la politique économique et sociale
}

Isabelle Bourgeois

\section{OpenEdition}

\section{Journals}

Édition électronique

URL : http://journals.openedition.org/rea/205

DOI : $10.4000 /$ rea.205

ISBN : 978-2-8218-0842-3

ISSN : 1965-0787

Éditeur

CIRAC

Édition imprimée

Date de publication : 1 octobre 2005

Pagination : 7-18

ISSN : 1156-8992

Référence électronique

Isabelle Bourgeois, « Années Schröder : les 'recentrages' successifs de la politique économique et sociale », Regards sur l'économie allemande [En ligne], 73 | octobre 2005, mis en ligne le 03 juillet 2008, consulté le 01 mai 2019. URL : http://journals.openedition.org/rea/205 ; DOI : 10.4000/rea.205 


\title{
Années Schröder : les 'recentrages' successifs de la politique économique et sociale
}

\author{
Isabelle Bourgeois
}

Durant ses 7 ans à la tête de deux gouvernements de coalition SPD/Verts, le chancelier Gerhard Schröder aura fini par s'imposer comme celui qui a commencé à rompre avec l'inertie. Mais il n'a engagé les réformes que sous la contrainte, et très tardivement. Les programmes Avenir 2000 et surtout Agenda 2010 n'ont été présentés qu'en réaction à l'évolution du contexte extérieur et à la menace d'un blocage intérieur. Dans la genèse des décisions, les experts auront joué un rôle fondamental, à l'instar du Conseil des Sages qui n'a cessé d'identifier les priorités et de formuler des recommandations d'action dont s'est largement inspiré le gouvernement. De leur côté, les partenaires sociaux ont mené une politique de rénovation de la régulation du travail qui a été non seulement conséquente, mais qui a aussi largement contribué à donner pleinement leur sens au cap gouvernemental des réformes. Ils se sont révélés par là des acteurs politiques à part entière.

Si la politique de Gerhard Schröder n'a pas été exempte de tâtonnements ni de tentations dirigistes, le jeu institutionnel allemand a toujours fini par la ramener à l'orthodoxie, forçant le gouvernement à 'recentrer' sa politique pour ramener l'économie allemande sur la voie de la croissance et mettre son action en conformité avec la dynamique de l'intégration européenne. Si le chancelier a su valoriser opportunément ses réformes en assumant la responsabilité de l'Allemagne dans une Europe en quête de compétitivité, il a su aussi mettre à profit une mutation des valeurs qui avait fini par générer un terrain favorable au changement. Le marasme économique avait fait prendre conscience à l'opinion de l'urgente nécessité d'amorcer des réformes structurelles. Petit à petit, une sorte de 'nouveau réalisme' s'est ainsi fait jour outre-Rhin. II permet aujourd'hui d'aborder plus ouvertement les problèmes et d'en considérer les causes profondes: l'Allemagne n'est pas victime de la globalisation; c'est bien au contraire son propre modèle économique et social qui lui interdit de développer une croissance génératrice d'emplois. Mais du diagnostic au traitement, la voie est longue. Les véritables réformes n'ont été lancées qu'en 2003, et elles restent largement inachevées. "Nous avons bien trop tardé ", avouait le chancelier lors d'une conférence de presse (29-08-2004). Surtout, les deux gouvernements SPD/Verts aux affaires depuis 1998 ont longtemps mené une politique peu lisible et même contradictoire avant d'oser le changement...

La politique économique et sociale menée de 1998 à 2005 par la coalition fédérale SPD/Verts marque le début d'un tournant vers la modernisation du «modèle rhénan ». Mais ce tournant a été durement négocié : le gouvernement ne l'a pris que sous la contrainte des réalités économiques, avant de s'y engager résolument et de prôner l'acceptation du changement. Les jalons de ce retour au réalisme ont été posés à deux reprises : entre 1999 et 2001, durant le premier mandat du gouvernement Schröder, puis à partir de 2003, au milieu du second. Dans les deux cas, on observe un scénario similaire : les experts et les milieux économiques, conscients des enjeux européens et mondiaux de la

Deux tournants : 1999/2001 et 2003 
1999 : retour au réalisme avec Hans Eichel

Avenir 2000 : annonce de réformes et vote des " retraites Riester"

compétitivité du site Allemagne, ont forcé la coalition à réviser sa politique initiale pour en rendre le cap plus lisible et, surtout, pour tenter de le 'recentrer' afin de débrider la dynamique de croissance. Ce sont eux qui, en plaidant patiemment pour les réformes, ont fini par convaincre le monde politique de la nécessité d'en revenir à l'orthodoxie de l'économie sociale de marché : en l'occurrence, à rompre avec la dérive distributive d'un Etat providence qui inhibe à la fois l'offre et la demande, creuse la dette publique et entretient un niveau de chômage élevé.

\section{Schröder I : un cap réaliste entre 1999 et 2001}

Un premier cap illisible et contradictoire nomiques grâce à une politique en trois volets (voir REA 59/02). Le premier: l'annonce d'une spectaculaire compression des dépenses publiques rassure les partenaires européens quant à la volonté de l'Allemagne de respecter le Pacte de Maastricht. La seconde mesure vise à rassurer les investisseurs internationaux : c'est l'annonce, faite à la veille de Noël 1999, de défiscaliser les plusvalues de cessions réalisées par la banque et l'assurance ; elle signifie la prochaine modernisation de la Deutschland AG (grâce au 'détricotage' du lien banque-industrie). Elle sera suivie d'une réforme fiscale allégeant les prélèvements sur les revenus moyens (entreprises et particuliers) et permettant une plus grande fluidité des capitaux (voir REA 54/01). Désormais, c'est Hans Eichel qui incarnera le retour au réalisme d'un premier gouvernement Schröder qui, après avoir donné des gages à son partenaire les Verts, renoue avec la tradition orthodoxe de la social-démocratie.

Le troisième volet consiste dans le lancement d'un programme Avenir 2000 annonciateur de réformes structurelles : modernisation de l'administration, réforme de l'Etat-providence, poursuite de la fiscalité écologique («écotaxe »). C'est dans ce cadre qu'est adoptée la " réforme Riester » qui introduit des éléments de capitalisation dans le régime légal des retraites par répartition. Mais la nouvelle approche traduit aussi la quête d'un savant équilibre entre éléments d'offre et de demande, cherchant à concilier les approches 'de gauche' et un pragmatisme 'libéral'. Car s'ajoutent à ces mesures des contreparties dans le domaine social : instauration du droit au temps partiel pour tout salarié qui le désire, ou réforme de la loi sur la Constitution interne de l'entreprise (abaissement à 5 salariés du seuil à partir duquel les salariés élisent leur Conseil d'établisse- 
ment ; voir REA 50/01). II est vrai que la croissance est au rendez-vous en cette fin de siècle et semble permettre quelques marges de manœuvre.

Ensuite, l'ardeur réformatrice du gouvernement Schröder I fléchit de nouveau, face à d'importantes échéances dans le calendrier électoral des Länder et l'approche des élections au Bundestag. Le retour à l'inertie et à ce que le chancelier appelle une "politique de la main tranquille », mais où les milieux économiques décèlent plutôt un cap de «navigation à vue », marquent la fin de ce premier mandat. Les dissensions politiques internes aux partenaires de la coalition ont repris le dessus, de même que les stratégies électorales menées par les partis dans les élections parlementaires des Länder. S'y ajoute la dégradation du contexte conjoncturel. L'éclatement de la bulle spéculative sur les valeurs technologiques a mis fin à l'illusion d'une croissance quasi automatique, et la hausse brutale du chômage comme la faiblesse de la consommation n'incitent guère non plus aux réformes structurelles. Mais après une année 2001 où l'économie allemande frise la récession, quelques signes annonciateurs de reprise se manifestent au début de 2002. A l'approche des élections au Bundestag, ces évolutions amènent le gouvernement à abandonner son cap de rigueur budgétaire, quitte à ne plus respecter le Pacte de Maastricht.

La politique du premier gouvernement Schröder se caractérise ainsi par une certaine propension à vouloir concilier des approches contradictoires et à expérimenter une 'autre' politique. II faut dire que le contexte de croissance de la fin des années 1990 lui donnait encore une manière de sursis dans le traitement des problèmes structurels. D'un autre côté, il n'avait pas la pleine marge de manœuvre pour mener une politique plus proactive. En 1998, face au chancelier Kohl, le candidat Schröder avait défendu un projet de « nouveau centre », c'està-dire d'un libéralisme rénové. Mais avec 298 sièges seulement sur les 669 que comprend à l'époque le Bundestag, le SPD ne réalise qu'un score modeste et peine à affirmer son leadership à gauche. Pour gérer l'alternance, il s'accorde avec le parti Bündnis 90/ Die Grünen (47 sièges) ; la coalition a une courte majorité de dix sièges au Bundestag. Gerhard Schröder doit alors composer doublement pour gouverner : avec l'aile gauche de son propre parti (représentée par Oskar Lafontaine) et avec son partenaire Vert. Ces contraintes expliquent l'adoption d'un cap de « renouveau écologique » dont la première manifestation sera l'abandon du nucléaire (signé avec la branche le 15-06-2000). II se traduira ensuite par la création d'un «impôt écologique » (écotaxe) frappant la consommation d'énergie et destiné en partie à subventionner le développement des énergies renouvelables.

Si le SPD a fait un pas en direction des Verts sur le nucléaire, ceux-ci ont commencé à découvrir le dossier du financement de la protection sociale et de l'impact des prélèvements sur les coûts salariaux. Il est vrai que dans la culture des Verts, le concept de «développement durable » va bien au-delà des préoccupations environnementales. II prolonge la définition née à l'origine dans la sylviculture prussienne du XVIIle siècle, en y ajoutant le concept d'équité intergénérationnelle. Mais dans un premier temps, cette notion reste teintée d'idéologie écologiste, comme en atteste cette nouvelle recette fiscale qu'est l'écotaxe ; son produit, sous couvert de préoccupations environnementales, est en réalité destiné à combler partiellement le déficit des caisses de retraites afin d'éviter dans l'immédiat la hausse des prélèvements et ses effets dommageables sur l'emploi (voir REA 48/00). Ainsi que le formulait à l'époque Otto Schlecht, exsecrétaire d'Etat au ministre fédéral de l'Economie sous diverses majorités, de 1962 à 1991, cette mesure fiscale " camoufle le coût réel de l'assurance retraite et retarde une réforme conséquente du système des retraites » (Handelsblatt, 21-09-2000). Ce nouvel impôt a aussi, soit dit en passant, l'avantage plus immédiat de lisser en partie les effets de la réforme fiscale.

Personne, dans la coalition SPD/Verts, n'établit alors encore ouvertement le lien entre le poids des prélèvements et la croissance ou, plutôt, la compétitivité.
Puis « politique de la main tranquille»

La difficile cohérence de la majorité 'plurielle'

Objectifs 'fourre-tout' de l'écotaxe

Le lien entre prélèvements et compétitivité n'est pas affiché 
Cette question reste réservée aux experts (le Conseil des Sages y avait consacré son rapport annuel 1997/98) ou est considéré comme faisant partie du fonds idéologique de l'Union chrétienne-démocrate et des libéraux du FDP, autrement dit : du précédent gouvernement, mené par Helmut Kohl, qui avait publié en 1993 un rapport conséquent sur la compétitivité future du site Allemagne (voir Commissariat Général du Plan/DFI, 2001). Il faut rappeler aussi que l'euphorie et la croissance de la "nouvelle économie » masquent les causes structurelles, domestiques, d'un potentiel de croissance sur le déclin. Et si l'Union économique et monétaire existe sur le papier (elle a été conclue le 2 mai 1998), elle ne produit pas encore ses effets au sein du marché unique en faisant apparaître les disparités structurelles dans la compétitivité des Etats membres. Celles-ci ne se révéleront réellement dans leur ampleur qu'après l'élargissement de l'UE (mai 2004).

Cependant, dans un espace communautaire de plus en plus intégré, l'approche globale allemande de la compétitivité trouvera son prolongement naturel dans les objectifs conjoints définis lors du Sommet européen de Lisbonne en 2000 : libérer la dynamique de croissance (et d'innovation), source d'emplois, pour faire de l'UE une économie compétitive dans l'économie du savoir et de la connaissance (voir REA 61/03). Mais l'action du premier gouvernement Schröder se concentrera sur l'innovation et, dans une moindre mesure seulement, sur des champs liés à l'environnement réservé aux activités ou à l'attractivité du site : baisse de la charge fiscale, notamment sur les PME, défiscalisation des plus-values, "réforme Riester » qui vise à réduire la part du financement fiscal des retraites en introduisant des éléments de capitalisation (voir REA 70/05). Cette première législature laisse ainsi un bilan en demi-teinte où l'ardeur réformiste aura été de courte durée, précédée d'une phase d'hésitation et d'expérimentation brouillonne, et suivi d'un cap de navigation électoraliste.

\section{Schröder II : un programme de réformes structurelles en 2003}

Septembre 2002-mars 2003 : de longs mois d'indécision

Rien, dans le contexte politique, n'incitait au changement
Ce n'est qu'au cours de son second mandat, à partir de mars 2003, que le gouvernement Schröder se réclamera ouvertement de la Stratégie de Lisbonne et déclinera les différentes facettes de cet objectif communautaire en domaines de politique nationale. Ce sera l'Agenda 2010 dont le chancelier décrira ainsi l'approche dans la brochure d'information destinée à l'opinion : "Le politique crée les conditions préalables au changement nécessaire. Mais il faut que l'ensemble de la société y apporte sa contribution ». C'est bien d'un processus global qu'il s'agira, contrairement à l'impression que donne au premier abord le catalogue de mesures annoncées en mars 2003. Mais avant de se résoudre au changement - contraint et forcé par la hausse continue du chômage, la récession et l'avertissement de la Commission quant à la dégradation des finances publiques, sans oublier les déconvenues successives de son parti dans les Länder -, le chancelier aura longtemps atermoyé.

En 2002, la constellation politique est en effet favorable au statu quo. Certes, lors des élections, le SPD ne bat I'Union CDU/CSU menée par Edmund Stoiber que d'un peu plus de 6000 voix. Donné longtemps battu d'avance dans les sondages, Gerhard Schröder doit son avantage bien plus à son aura médiatique personnelle qu'à son programme. Plaidant pour "le renouveau dans la cohésion ", c'est-à-dire pour une politique de réformes prudentes ne heurtant aucune sensibilité au sein d'un parti qu'il était parvenu à unir derrière lui, il incarnait aussi un certain style de vie ouvert au monde et hédoniste - plus séduisant aux yeux de quelques poignées d'électeurs que la compétence économique reconnue, mais considérée comme 'socialement' froide, d'un Edmund Stoiber qui prônait des réformes plus radicales. Cette très courte avance amène le chancelier à reconduire la coalition SPD/Verts. Elle ne dispose certes que d'une majorité de 3 sièges au Bundestag ; mais à l'époque, la moitié des Län- 
der est gouvernée par le SPD, seul ou en coalition. L'opposition au Bundesrat étant faible, rien n'incitait donc au changement.

Dans cette situation politiquement 'confortable', les partis de coalition ont pris leur temps pour ajuster leur programme. Les Verts, en position de force puisqu'ils avaient été reconduits à l'échelon fédéral (ce qu'ils doivent à leur très médiatique chef de file Joschka Fischer) et qu'ils participent à plusieurs coalitions dans les Länder, contribueront largement à la définition d'un texte qui relève plus d'un catalogue de bonnes intentions que d'un projet de nature à sortir l'économie allemande de la récession où elle semble vouloir s'installer. Ce programme, intitulé «Renouveau-Justice-Développement durable », laisse pantois les milieux économiques, d'autant que la première mesure du gouvernement sera d'augmenter les impôts et les cotisations. Confrontées dans le même temps à la flambée des prix pétroliers due à la crise irakienne et à l'appréciation de l'euro face au dollar, les entreprises préfèrent accroître productivité et taux d'utilisation des capacités plutôt que d'investir et d'embaucher. Quant aux ménages qu'inquiète déjà la situation sur le marché de l'emploi, ils entament une "grève de la consommation ».

Très vite, ce gouvernement perd la confiance des milieux économiques. Instituts de conjoncture et Conseil des Sages ne mâchent pas leurs mots quant à l'incohérence du cap présenté. Dans leur rapport d'automne 2002, les premiers incriminent plus particulièrement l'abandon de la politique de la politique de rigueur: "il ne suffit pas de se contenter d'annoncer un objectif de consolidation ", écrivent-ils, rappelant qu'il convient plutôt d'identifier les subventions à réduire et de définir les réformes à mener pour ramener les transferts sociaux à de plus justes proportions. Leur seconde critique porte sur les travaux de la Commission Hartz, instituée au printemps 2002, dont les propositions «ne peuvent pas tenir lieu de traitement de fond du chômage ». II conviendrait plutôt de créer un environnement favorable, une mission qui relève des pouvoirs publics comme des partenaires sociaux, non pas d'une commission créée originellement pour restructurer l'Office fédéral du Travail de Nuremberg (aujourd'hui rebaptisé Agence fédérale pour l'emploi).

Quant au Conseil des Sages, il rappelle le gouvernement à son devoir dans son rapport annuel 2002/03 publié en novembre 2002. II y détaille entre autres les problèmes structurels du marché de l'emploi qui sont le principal frein à la compétitivité allemande : 'rigidités' dues au cadre réglementaire et poids des prélèvements. Et il rappelle que, loin de s'attaquer à la racine du mal, le premier gouvernement Schröder a contribué à aggraver la situation. Plus généralement, le Conseil des Sages pointe du doigt une série d'entorses à l'orthodoxie du « pilotage global » (voir encadré) et, point essentiel, les manquements à la réalisation des deux objectifs prioritaires dans ce contexte que sont la croissance et l'emploi. Pour le guider dans son action, il formule une série de recommandations : ces « 20 points pour la croissance et l'emploi » qui sont également le titre du rapport (Bourgeois, 2005). Conscients des résistances que ne manqueront pas de susciter celles qui visent l'emploi (baisse des prélèvements, modération salariale et abaissement du seuil des salaires minimum implicites de référence), les Sages délivrent ce message : «le retour au plein emploi implique des sacrifices en termes de redistribution et d'Etat-Providence ».

Les préconisations du Conseil des Sages ne sont certes pas contraignantes quant à la méthode, mais cette instance créée en 1963 et composée de personnalités indépendantes est une sorte de gardien de l'orthodoxie. Elle a pour mission d'évaluer l'évolution macro-économique, de formuler une critique constructive à l'encontre de la politique gouvernementale et, en cas de dysfonctionnements constatés dans l'évolution économique et sociale, de formuler les grandes orientations d'une politique de réformes. Le gouvernement fédéral suivra ses recommandations : elles inspireront largement l'Agenda 2010. Une fois encore, le réalisme économique reprendra alors ses droits.
Un programme gouvernemental inconsistant...

... vivement critiqué par les Instituts de conjoncture

Le rappel à l'ordre du Conseil des Sages...

... et l'Agenda 2010 en réaction 


\section{Principaux champs d'action des gouvernements Schröder (1998/2002 et 2002/05)}

\begin{tabular}{|c|c|}
\hline Budget & $\begin{array}{l}1998 \text { (ministre fédéral des Finances : O. Lafontaine) : hausse de } 6 \% \text { des dépenses publiques et politique de } \\
\text { relance de la consommation des ménages (hausse des allocations familiales, baisse de l'impôt sur les } \\
\text { revenus modestes); } \\
1999 \text { (Hans Eichel) : retour à l'austérité budgétaire et policy mix mêlant éléments d'offre et de demande } \\
\text { dans le domaine social ; } \\
2002 \text { : à l'approche des élections au Bundestag, H. Eichel promet de ramener le déficit en dessous de la } \\
\text { barre des } 3 \% \text { d'ici } 2004 \text { et écarte ainsi la menace de sanctions communautaires ; mais le gouvernement fé- } \\
\text { déral assouplit quelque peu sa discipline budgétaire (notamment par l'allégement de la fiscalité des revenus } \\
\text { moyens), et le déficit allemand s'établit à } 3,7 \% \text { à la fin de l'année. Ensuite, le déficit continue de se creuser, } \\
\text { les Länder refusant d'adopter au Bundesrat le projet de contrefinancement des allégements fiscaux par la } \\
\text { suppression d'une série de subventions et de niches fiscales. En } 2006 \text {, pour la } 5^{\mathrm{e}} \text { année consécutive, } \\
\text { l'Allemagne ne respectera pas les critères de Maastricht. } \\
\text { Depuis 1998, la politique budgétaire du gouvernement fédéral oscille entre austérité et éléments de relance } \\
\text { de la consommation. Le dernier aspect gagne en importance depuis que la conjoncture allemande s'est ins- } \\
\text { tallée dans le marasme. Les difficultés liées au financement de l'unification, argument majeur présenté lors } \\
\text { des négociations sur un assouplissement du Pacte de stabilité et de croissance ne sont pas la seule cause } \\
\text { de la dérive budgétaire allemande, entretenue aussi par la faible discipline budgétaire des Länder et un défi- } \\
\text { cit croissant des systèmes de protection sociale que peut seulement comprimer le recours à une fiscalisation } \\
\text { accrue de leur financement. }\end{array}$ \\
\hline Fiscalité & $\begin{array}{l}1999 \text { : lancement de la « réforme fiscale écologique ». L'écotaxe entre en vigueur en } 3 \text { étapes (1999, } 2001 \\
\text { et } 2002 \text { ). Elle a pour objectif de renchérir la consommation d'énergie, son produit est destiné à la fois à fi- } \\
\text { nancer le développement d'énergies renouvelables et à comprimer la hausse des prélèvements retraites afin } \\
\text { de favoriser l'emploi ; } \\
2000 / 03 \text { : réformes de la fiscalité. Harmonisation des régimes fiscaux de l'IR des personnes physiques et } \\
\text { morales ; élargissement de l'assiette de l'IR et baisse des taux d'imposition aux deux extrémités (minimum : } \\
15 \% \text {; maximum : } 42 \% \text { ). Le taux de l'IS est abaissé à } 25 \% \text { et quelques 'niches' sont abolies en attendant } \\
\text { une harmonisation européenne de la fiscalité des entreprises ; maintien d'un régime plus favorable pour les } \\
\text { compagnies d'assurance, acteurs majeurs dans la réforme des retraites (capitalisation). } \\
\text { Depuis 1998, la politique fiscale du gouvernement Schröder s'est contentée de baisser les prélèvements } \\
\text { pour soutenir la consommation et les activités des PME, mais tout comme les gouvernements Kohl, elle a } \\
\text { éludé la question de fond: le poids des prélèvements pour les entreprises et, plus généralement, l'opacité } \\
\text { du code fiscal allemand. }\end{array}$ \\
\hline Emploi & 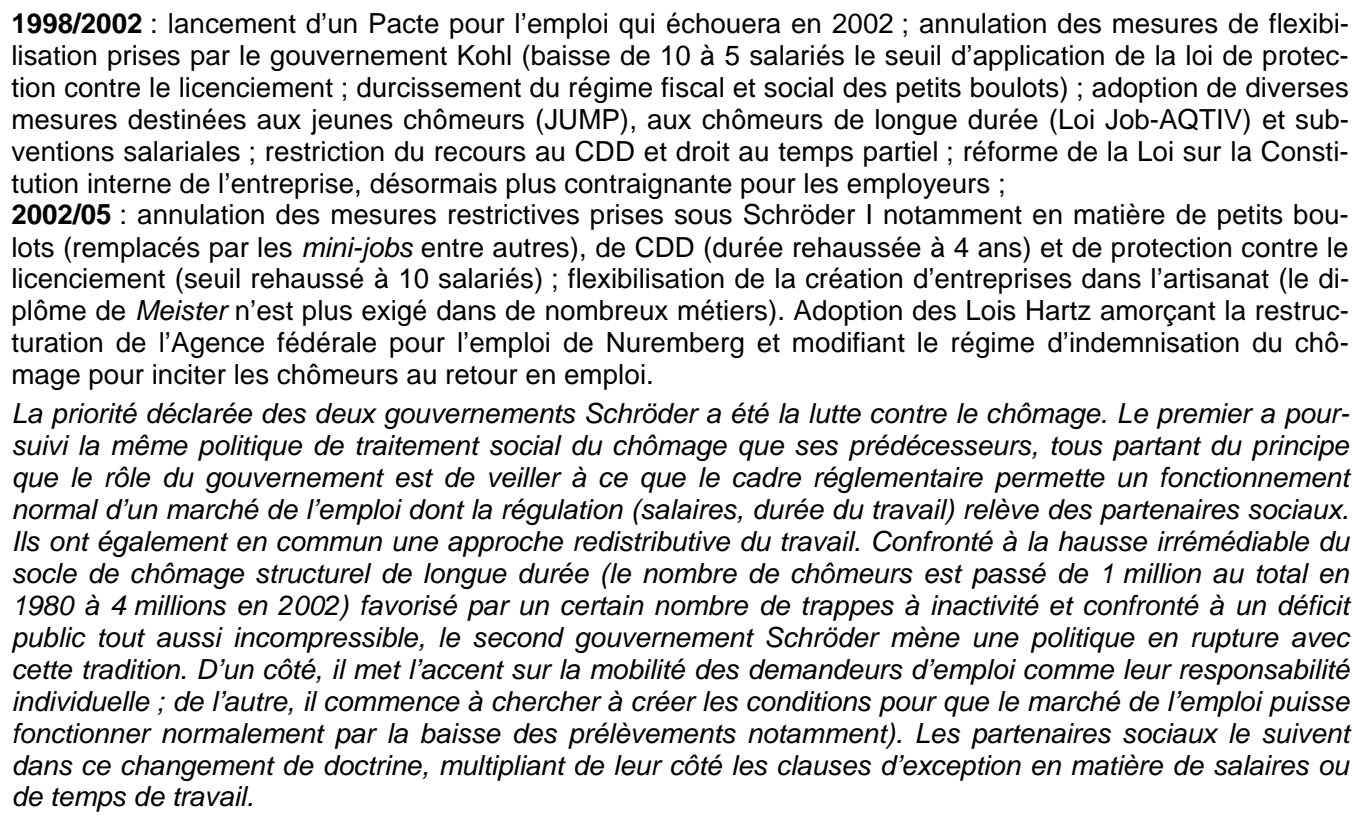 \\
\hline Santé & $\begin{array}{l}1998 \text { : abolition des mesures prises par le gouvernement Kohl (à savoir la hausse de la participation finan- } \\
\text { cière des assurés à certains soins et prestations); compression des dépenses de médicaments; } \\
2003 \text { : hausse de la participation financière des assurés (taxe de cabinet médical, hausse des tickets modé- } \\
\text { rateurs et réduction de la prise en charge de certaines prestations) ; meilleure coordination des soins ; for- } \\
\text { mation continue obligatoire pour les médecins. } \\
\text { Après un premier cap dédié à la “ justice sociale ", le déficit croissant des caisses d'assurance maladie et la } \\
\text { hausse incontournable des prélèvements aboutissent à des inflexions. La priorité du second gouvernement } \\
\text { Schröder est désormais la compression des prélèvements, entre autres grâce à une fiscalisation accrue du } \\
\text { financement de la protection maladie et à l'abandon du financement paritaire : désormais, la part employeur } \\
\text { s'élève à } 6,65 \% \text {, la part du salarié (et des retraités) s'élève à } 7,55 \% \text { du salaire brut. }\end{array}$ \\
\hline
\end{tabular}




\begin{tabular}{|c|c|}
\hline Retraites & 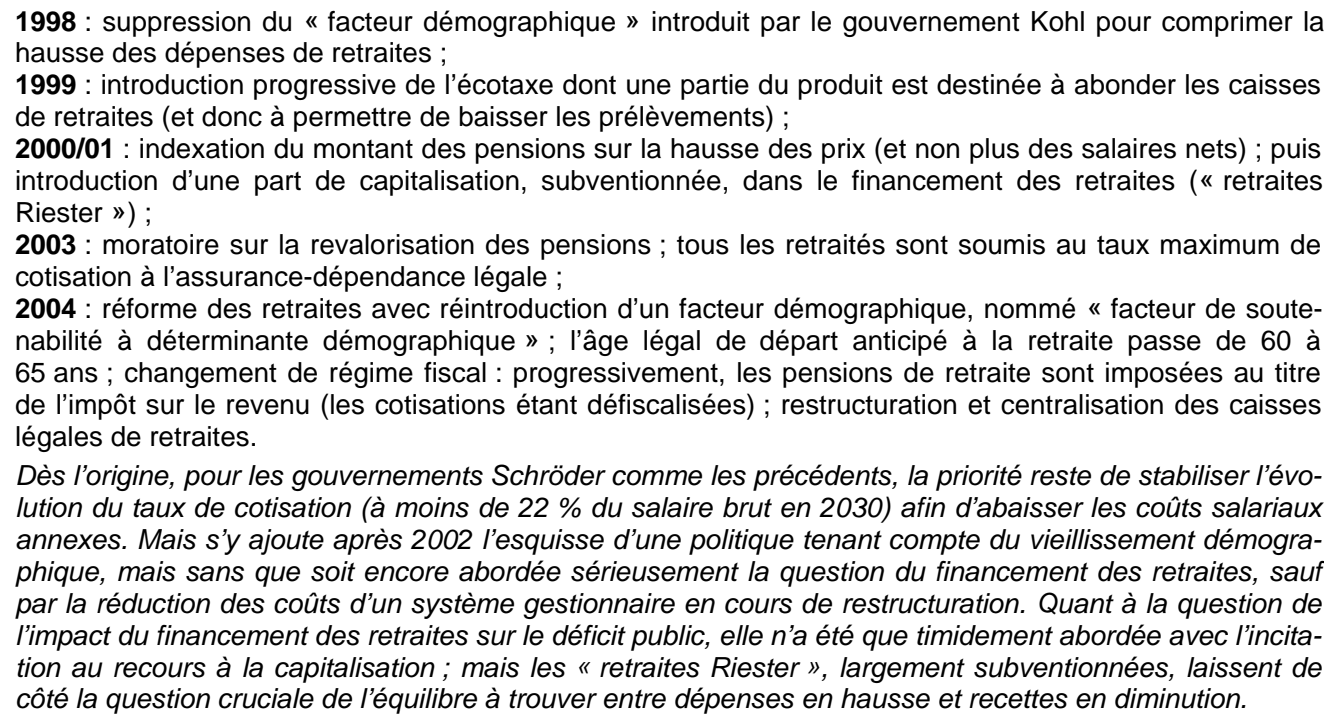 \\
\hline Famille & 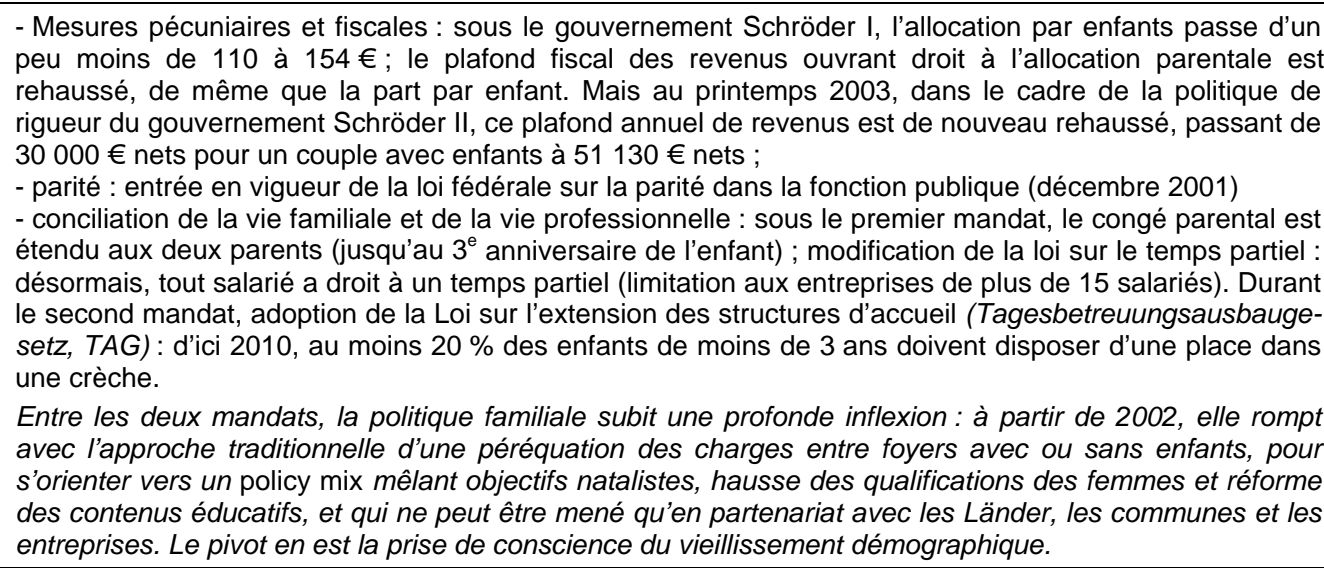 \\
\hline $\begin{array}{l}\text { Energie/ } \\
\text { Environnement }\end{array}$ & $\begin{array}{l}\text { La première mesure du gouvernement Schröder I est l'abandon progressif du nucléaire à l'horizon } 2022 \text {, } \\
\text { puis le développement des énergies renouvelables, subventionnées notamment grâce à l'écotaxe. En } \\
\text { contrepartie de ce programme imputable aux Verts, le SPD programme la poursuite de l'exploitation, } \\
\text { subventionnée, des gisements de houllle jusqu'en } 2012 \text {. Sous le gouvernement Schröder II, la } \\
\text { libéralisation des marchés du gaz et de l'électricité se poursuit, en application des directives européennes. } \\
\text { En matière d'environnement, la principale mesure est l'adoption d'une consigne sur les canettes de } \\
\text { boissons, entrée en vigueur en mai } 2005 \text {. } \\
\text { Si les Verts ont pu imposer leur projet d'abandon du nucléaire et d'un approvisionnement énergétique } \\
\text { reposant largement ( } 20 \% \text { en 2020) sur les énergies renouvelables, les gouvernements Schröder ne sont } \\
\text { pas parvenus à définir les orientations d'un nouvel Energiemix qui soit à la fois compatible avec son projet } \\
\text { d'une " modernisation écologique de la société industrielle", les contraintes du Protocole de Kyoto et les } \\
\text { besoins d'une économie compétitive. }\end{array}$ \\
\hline Innovation & $\begin{array}{l}\text { Comme pour le gouvernement Kohl dès le milieu des années } 1990 \text {, la promotion des technologies-clefs } \\
\text { (nanotechnologies, biotechnologies, TIC) et de l'innovation constitue une priorité pour les deux gouverne- } \\
\text { ments Schröder. Cette priorité, qui découle en partie de l'engagement communautaire (PCRD), est renfor- } \\
\text { cée depuis le Conseil de Lisbonne en } 2000 \text {. Elle se traduit de deux manières : par la hausse des moyens } \\
\text { consacrés à la R\&D (elle dépasse actuellement } 2,5 \% \text { du PIB) et, surtout, par une politique d'offre où les } \\
\text { pouvoirs publics cherchent à améliorer le contexte des activités privées. L'approche (bottom up) est celle } \\
\text { d'une compétitivité globale générée par l'interaction systématique entre initiative privée et action publique } \\
\text { à tous les échelons territoriaux. Le gouvernement fédéral se contente de tenir à disposition des crédits et } \\
\text { des mécanismes de soutien (surtout la communication), mais ne définit pas de pôles de compétitivité : le } \\
\text { soutien ciblé de ces pôles, créés par la constitution de grappes (clusters) générées par un processus } \\
\text { d'interpénétration de l'activité des entreprises locales, des universités et des laboratoires de recherche } \\
\text { appliquée, relève en effet de la souveraineté des Länder en matière de politique économique et } \\
\text { d'innovation (voir REA } 57 / 02,61 / 03,64 / 03,66 / 04 \text { et } 72 / 05 \text { ). } \\
\text { La politique de la coalition fédérale est néanmoins marquée par des différences d'approche. Alors que le } \\
\text { chancelier promeut très activement l'innovation et la recherche avec son " offensive pour l'innovation ", } \\
\text { cherchant à impulser dans l'opinion une culture favorable, les Verts se concentrent sur les questions } \\
\text { éthiques, particulièrement dans les sciences du vivant. C'est ainsi que la loi de } 2002 \text { autorise la recherche } \\
\text { sur les cellules embryonnales - à condition que celles-ci soient importées. De même, les Verts (ministère } \\
\text { fédéral de l'Agriculture) sont parvenus à limiter la recherche en matière d'OGM. }\end{array}$ \\
\hline
\end{tabular}


Les contraintes du fédéralisme..

forcent aussi à la construction d'équilibres

Un fonds culturel commun : l'ordo-libéralisme

Depuis 2000, l'Allemagne est la « lanterne rouge de l'Europe"
Les évolutions du rapport de forces entre échelons du Bund et des Länder contribueront elles aussi à forcer le 'recentrage' de la politique du gouvernement fédéral. Au gré des élections parlementaires dans les Länder, aussi bien le SPD que les Verts sont placés progressivement dans l'opposition. La représentation de l'exécutif régional à l'échelon fédéral, le Bundesrat, est maintenant dominée par l'Union CDU/CSU. Cela aboutit à une sorte de cohabitation politique qui exige la négociation de compromis pour définir une politique consensuelle, puisque le processus législatif exige le vote conjoint des deux chambres dans la plupart des domaines. Tel fut le cas de la loi Hartz IV (voir REA 68/04).

Mais cette situation a aussi souvent été source d'immobilisme. Car les Länder défendent leur propre politique budgétaire, économique et d'innovation; au Bundesrat, ils le font avec d'autant plus d'acharnement que, au fil de l'histoire du fédéralisme allemand, s'est dégagée une centralisation rampante des pouvoirs au profit de l'échelon fédéral. C'est ainsi que l'indispensable réforme institutionnelle du fédéralisme a achoppé cette année sur la question des compétences en matière de politique universitaire. En matière budgétaire et fiscale, elle avait été ajournée à... 2019 sous le premier gouvernement Schröder.

Or s'ils peuvent durer longtemps, ces blocages institutionnels ne sont jamais insurmontables. Ainsi, la réforme fiscale projetée par le gouvernement Kohl s'était heurtée pendant près de dix ans à l'obstruction d'un Bundesrat où dominaient alors les Länder dirigés par un gouvernement SPD (ses principaux détracteurs étaient le ministre-président de Sarre, Oskar Lafontaine, et son homologue de Basse-Saxe, Gerhard Schröder). Elle n'aboutira qu'en juillet 2000 (voir REA $54 / 01$ ), sous le premier gouvernement Schröder; depuis, le rapport des forces s'était inversé au Bundesrat et les différents projets en concurrence avaient pu être ajustés. La structure institutionnelle de la RFA fait ainsi que la politique économique et sociale du gouvernement fédéral est toujours le fruit d'une lente construction d'équilibres respectant les intérêts des divers courants de l'opinion (via leur représentation politique), des différents échelons territoriaux comme des intérêts organisés de la société civile (corps intermédiaires, dont les syndicats et leurs homologues patronaux). II n'en va pas autrement en 2005.

Quels que soient les projets de l'une ou l'autre des formations politiques, dans la pratique, elles doivent non seulement concilier ces différents intérêts, mais aussi respecter un subtil dosage entre une politique économique destinée à libérer les forces du marché et une politique sociale menée en correctif de la loi du marché. Certes, les différents partis ont des cultures idéologiques propres mais, à l'exception du PDS d'obédience marxiste comme du récent rassemblement d'extrême gauche Linkspartei, ils se rejoignent sur la vision partagée d'un libéralisme organisé (économie sociale de marché). Autrement dit : par-delà les mécanismes institutionnels qui forcent au consensus, les forces politiques se réclament toutes (sauf la Linkspartel) de la même culture du marché et de la performance économique. C'est donc aussi l'existence de ce dogme commun (voir encadré) qui avait forcé le gouvernement Schröder I à 'recentrer' sa politique et amené le second à présenter son Agenda 2010 le 14 mars 2003.

Dès la fin de la première législature, la conjoncture allemande s'est dégradée. L'éclatement de la bulle spéculative sur les valeurs technologiques, puis le ralentissement de l'activité mondiale avaient plongé l'économie nationale dans un marasme persistant qui fait de l'Allemagne la «lanterne rouge de l'Europe ». La formule est popularisée outre-Rhin en décembre 2002 par Hans-Werner Sinn, le très médiatique patron de l'institut ifo. La consommation intérieure reste structurellement le point faible de l'économie. L'Allemagne ne respecte plus les critères de Maastricht depuis trois ans. Seules les performances à l'export permettent d'éviter la récession; mais l'industrie allemande n'a pu maintenir sa compétitivité internationale qu'au prix d'une modération salariale unique en Europe et de la compression des effectifs sur le sol allemand. Le socle structurel du chômage s'élève irrémédiablement ; aucune des mesures de traitement so- 
cial menées depuis le premier choc pétrolier et renforcées par le gouvernement Schröder I ne parvient à enrayer la tendance.

\section{Une doctrine commune qui favorise une politique 'centriste' : l'ordo-libéralisme}

Bien avant que le père du « miracle économique », le chrétien-démocrate Ludwig Erhard, ministre de l'Economie du chancelier Adenauer (1957-63) puis chancelier (1963-66) de la RFA, lance la notion de Soziale Marktwirtschaft (la traduction correcte en est : économie de marché sociale), la doctrine d'un capitalisme à dimension sociale avait été développée en Allemagne à la fin du XIXe siècle par des personnalités de toute obédience, en rupture à la fois avec le socialisme naissant et le 'laisser-faire' de l'Ecole de Manchester. En RFA, le parti SPD recréé après la guerre s'est rallié à cette doctrine lors de son congrès de Bad Godesberg (1959). C'est ce fonds commun qui permettra la constitution d'un gouvernement de 'grande coalition' rassemblant chrétiens-démocrates et sociaux-démocrates entre 1966 et 1969.

\section{7 : Loi de promotion de la stabilité et de la croissance}

L'approche commune du libéralisme organisé débouchera, en matière de politique, sur la doctrine du « pilotage global » ou de «l'action concertée » développée en 1967 par le ministre fédéral de l'Economie Karl Schiller (SPD). Elle se résume à cette formule : «Concurrence autant que possible ; planification juste autant que nécessaire ". Elle signifie que l'Etat fédéral se contente de donner des impulsions au plan macro-économique; tout le reste relève des lois du marché et des acteurs privés. Si des dysfonctionnements sont constatés dans la réalisation des objectifs gouvernementaux - mais seulement dans ce cas -, le gouvernement doit les corriger.

Depuis la Loi de promotion de la stabilité et de la croissance de l'économie (Gesetz zur Förderung der Stabilität und des Wachstums der Wirtschaft) adoptée en 1967, l'action de tout gouvernement doit poursuivre quatre objectifs : stabilité des prix, taux d'emploi élevé, croissance, équilibre des échanges extérieurs. L'économie allemande, championne à l'export, réalise largement ce dernier. La mission de la stabilité des prix a été récemment conférée à la $\mathrm{BCE}$. Restent la croissance et l'emploi. C'est sur les dysfonctionnements dans la réalisation de ces deux objectifs que devait dès lors se concentrer l'action des gouvernements Schröder.

\section{Une doctrine constitutionnalisée par les Juges de Karlsruhe}

La doctrine de l'ordo-libéralisme avait certes été popularisée en RFA par l'ouvrage de L. Erhard: Wohlstand für alle : La prospérité pour tous, publié en 1957. Mais c'est le Tribunal Constitutionnel fédéral qui, au fil de sa jurisprudence cinquantenaire, lui conférera rang constitutionnel. La Loi fondamentale est certes muette sur l'ordre économique de la RFA, se contentant d'instaurer un "ordre constitutionnel libéral et démocratique " (art. 18). Or le qualificatif de « libéral » s'entend dans le sens des libertés démocratiques. Et c'est au nom de la garantie des droits fondamentaux du citoyen (qui dispose de la saisine directe) que la Cour a construit sa jurisprudence, passant du social à l'économique jusqu'à intégrer les deux.

Ce sont plus particulièrement six de ces droits qui ont déterminé les principes d'organisation de la société allemande avec, en leur cœur, l'équilibre entre droit individuel et responsabilité collective, entre intérêt particulier et intérêt général : l'égalité devant la loi (art. 3), la protection due à la famille (art. 6), la liberté de réunion et d'association (art. 8 et 9), la liberté de circulation et d'établissement (art. 11), du libre exercice de la profession (art. 12), et la garantie du droit à la propriété (art. 14). Les arrêts de la Cour de Karlsruhe ayant force de loi et étant immédiatement applicables, ils ont par exemple influé sur l'organisation de la régulation : l'autonomie des partenaires sociaux et la cogestion découlent directement de cette pondération entre particulier et collectif.

La garantie de ces droits est indissociable des fondements de l'ordre étatique tels que les stipule ensuite l'art. 20 de la Loi fondamentale : "La République fédérale d'Allemagne est un Etat fédéral démocratique et social ». C'est ainsi que les arrêts de Karlsruhe déterminent aussi certaines priorités politiques : par exemple la réforme de la fiscalité des retraites, imposée au gouvernement Schröder II au nom du principe de l'égalité devant la loi (progressivement, les pensions de retraites privées comme publiques seront soumises à l'impôt sur le revenu). Le 27-09-2005, le même principe a amené les Juges de Karlsruhe à débouter de leur plainte trois fonctionnaires retraités s'estimant lésés par le fait que la réforme des retraites avait abaissé le montant des pensions à $71,75 \%$ du dernier salaire (au lieu de $75 \%$ auparavant). Car le droit individuel qu'est l'égalité devant la loi doit être mis en relation avec l'intérêt général, en l'occurrence la consolidation budgétaire et l'objectif de préserver l'avenir des retraites. La construction du droit allemand exige elle aussi la quête permanente d'un équilibre et de la pondération des intérêts.

Conjugué à l'interprétation des droits fondamentaux, le rôle d'arbitre que joue le Tribunal dans les conflits opposant Etat fédéral (Bund) et Etats fédérés (Länder), vise également à garantir une certaine cohésion au sein de la fédération. En matière de politique budgétaire, par exemple, " la Fédération et les Länder doivent tenir compte de l'équilibre global de l'économie " (art. 109 al. 2). Voilà qui plaide aussi bien pour une politique de consolidation budgétaire que pour la réforme d'un fédéralisme financier dont la dérive égalitariste des mécanismes destinés à l'origine à assurer à la fois la compétition et la solidarité des collectivités inhibe désormais elle aussi la croissance allemande. Dans son arrêt du 11-11-1999, les Juges avaient rappelé aux collectivités territoriales le concept à la base de ce fédéralisme «coopératif »: "l'interdiction du nivellement », marquant ainsi la limite entre équité et égalitarisme.

Cette situation interdit désormais toute illusion sur d'éventuelles causes externes de la faible croissance structurelle allemande et force le gouvernement fédéral à affronter la réalité des mutations économiques, de la globalisation et du vieillissement démographique. Et à reconnaître que c'est en réalité le coût démesuré de l'Etat social qui bride la croissance : en creusant le déficit public
C'est le coût de l'Etat social qui obère la croissance... 
... en accaparant plus de $42 \%$ du PIB

G. Schröder affronte les syndicats et se brouille avec la base du SPD

Les partenaires sociaux flexibilisent...

... et tiennent compte des coûts salariaux annexes au détriment de l'investissement, en renchérissant le coût du travail par des prélèvements toujours plus élevés, ce qui gonfle un chômage structurel qui, en retour, pèse sur les budgets sociaux. Seules des réformes de fond peuvent y remédier. Mais il faudrait les mener dans tous les domaines à la fois : consolidation budgétaire, réforme du financement de la protection sociale, flexibilisation du marché de l'emploi, renforcement de la politique d'innovation, politique familiale, hausse des qualifications. C'est bien un traitement au long cours qui s'impose.

Après avoir commencé par abaisser la fiscalité, puis par inciter à l'épargneretraite (« retraites Riester») durant son premier mandat, le gouvernement Schröder aborde durant le second le chantier de l'assurance-maladie. Mais face à plus de 4 millions de chômeurs, il concentre ses efforts sur le plus urgent. Car le chômage est à la fois effet et cause des difficultés économiques ; de surcroît, il déstructure le lien social. Pour le dire autrement: l'Allemagne est devenue une économie sociale au détriment de son économie de marché. Ce déséquilibre s'accroît depuis trente ans : le poids des prélèvements fiscaux et sociaux nécessaires pour financer l'Etat providence a doublé depuis, il accapare désormais plus de $42 \%$ du PIB.

$\mathrm{Ne}$ pouvant agir directement sur les politiques salariales et la régulation des conditions de travail puisqu'ils relèvent de la politique contractuelle des partenaires sociaux, le gouvernement fédéral met alors à l'ordre du jour la nécessité d'une flexibilisation des salaires (surtout de l'ouverture de la fourchette vers le bas). Et il mène une double action. II fait pression sur les syndicats en réitérant publiquement sa menace de modifier une partie du dispositif réglementaire à la base du système de négociation collective s'ils se crispent sur le statu quo. L'affrontement creuse l'écart idéologique avec une partie du mouvement syndical et de la base du SPD ; ajouté aux réformes Hartz, il amènera un peu plus tard Gerhard Schröder à démissionner de la tête du parti (la direction en est confiée à Franz Müntefering) pour avoir les coudées plus franches dans sa politique de réformes.

Pourtant, de son côté, le mouvement syndical n'est pas en reste. En dépit de débats internes virulents, il apporte sa contribution au changement de cap. La réalité des restructurations industrielles amène les centrales syndicales à poursuivre à plus grande échelle une politique de modulation des salaires et du temps de travail qu'ils avaient commencé à expérimenter dans les nouveaux Länder depuis 1993, puis à mettre en œuvre sur l'ensemble du territoire à partir de 2002. A l'été 2004, des accords sur le retour aux 40 heures sont ainsi signés dans un certain nombre d'entreprises phares. Or leur caractère spectaculaire a relégué au second plan l'importance d'un accord signé à Pforzheim au printemps 2004 par IG Metall et son homologue patronal Gesamtmetall. En permettant aux entreprises prospères de déroger aux normes de branche pour préserver leur compétitivité, il rompt avec la doctrine qui voulait que seule une entreprise en difficulté pouvait se livrer à ce que les critiques de cet accord n'ont pas manqué de qualifier de « dumping salarial » (Schrœder, 2005).

Les partenaires sociaux ont posé les jalons de la flexibilisation et commencé à ouvrir la fourchette salariale vers le bas - à condition toutefois que cette politique reste l'exception. Conscients de leur responsabilité dans l'organisation de l'Etat social allemand, ils vont plus loin encore, intégrant désormais dans leur réflexion le facteur des coûts salariaux annexes (voir REA 72/05). Ponctuellement, ils la mettent en œuvre dans leur politique contractuelle, par exemple chez DaimlerChrysler ou VW (hausse du temps de travail sans compensation salariale, mais avec une offre de prestations sociales en contrepartie), ou en s'efforçant de développer les retraites d'entreprise, voire un régime de retraites d'entreprises au niveau de la branche (Metallrente, voir REA 70/05). Autrement dit: les centrales syndicales, IG Metall en tête, partagent aujourd'hui le diagnostic des économistes sur les freins à la compétitivité allemande, plaçant leur 
analyse dans une approche globale, macro-économique et macro-sociale, et plaident pour une refondation de l'Etat social allemand. C'est ainsi qu'IG Metall a organisé en avril 2005 un «Congrès sur l'Etat social », le premier du genre outre-Rhin (voir REA 72/05).

A son niveau de compétences réglementaires, et avant même de lancer son programme Agenda 2010 (il mènera à l'assouplissement de la protection contre le licenciement, à la libéralisation du travail intérimaire et aux lois Hartz), le gouvernement Schröder II a commencé par abolir diverses mesures prises lors de son premier mandat et qui avaient renforcé les rigidités du marché de l'emploi. Il allonge ainsi la durée maximale des CDD ou facilite l'exercice des petits boulots (mini-jobs entre autres). L'ensemble de ces mesures contribue de facto à flexibiliser la réglementation du travail et à permettre l'émergence, hors du champ de la négociation contractuelle de branche, d'un segment à bas salaires. Si les petits boulots ont pour effet de réduire le travail au noir et d'accroître le nombre d'actifs occupés, ils pèsent en revanche sur les systèmes de protection sociale, puisqu'ils ne sont généralement pas (ou alors dans une faible mesure) soumis à cotisations sociales. Mais cet inconvénient pèse peu encore en comparaison des progrès de flexibilisation réalisés en matière d'ouverture de la fourchette salariale vers le bas. Le syndicat des services ver.di ne s'y est d'ailleurs pas trompé, qui revendique désormais la création d'un salaire minimum légal. Comme s'il avait eu peur de son propre courage, le gouvernement fédéral a repris l'argument à son compte à l'approche des élections en Rhénanie du Nord-Westphalie.

Le second volet de la politique pour l'emploi du gouvernement Schröder II vise, sur la base des travaux de la Commission Hartz et au sein de l'Agenda 2010, à réformer le dispositif d'indemnisation du chômage. Pour accroître l'efficience du placement des demandeurs d'emploi, l'Agence fédérale pour l'emploi de Nuremberg engage sa restructuration ; pour accélérer la rotation chômage/emploi, les chômeurs sont incités à la mobilité géographique et professionnelle et doivent accepter tout emploi, même rémunéré à un niveau inférieur à leur dernier salaire ou aux normes en vigueur dans la branche. Enfin, la loi Hartz IV incite fortement les chômeurs de longue durée à se réinsérer : est désormais considéré comme demandeur d'emploi quiconque est capable de travailler plus de 3 heures par jour ; et le versement d'un revenu de substitution (un forfait de $345 €$ à l'ouest au minimum) est soumis à conditions : quête active d'un emploi, participation à des mesures de requalification, absence de patrimoine ou de revenus familiaux dépassant un certain seuil (voir REA 68/04).

Cette loi Hartz IV est de loin la plus significative des réformes menées par le second gouvernement Schröder. Rappelons qu'elle a été négociée avec les partenaires sociaux et adoptée par les deux chambres du parlement, autrement dit par la majorité comme l'opposition et l'ensemble des collectivités territoriales. La loi repose donc sur un profond consensus, quoi qu'en disent ses détracteurs qui se recrutent pour l'essentiel dans les camps extrêmes de l'opposition politique, donc loin du contrat social allemand. Car elle s'attaque de front aux trappes à inactivité qui excluaient du marché du travail et de la société quelque trois millions d'Allemands. Ce faisant, le chancelier poursuivait aussi un objectif de consolidation budgétaire : contribuer à comprimer le déficit structurel en réduisant les dépenses de l'assurance chômage. Indirectement, elle visait de surcroît à permettre aux communes de comprimer leurs dépenses d'aide sociale afin de leur restituer un peu de marge pour leurs investissements. Mais surtout, en revalorisant la valeur travail, c'est-à-dire en rappelant le nécessaire équilibre entre les droits et devoirs de chacun, entre l'intérêt particulier et l'intérêt général, Gerhard Schröder a amorcé un retour aux valeurs fondatrices du modèle social et économique allemand. Bien sûr, la loi Hartz IV ne crée pas d'emplois ; elle ne le peut pas, puisque ce n'est pas son objectif. Mais elle pose un des jalons indispensables dans le cadre d'un long processus global visant à rééquilibrer les deux termes du contrat social allemand: la compétitivité et la
Réglementation du travail : vers un segment à bas salaires

Réforme du dispositif d'indemnisation chômage

Hartz IV : retour aux termes du contrat social allemand 
solidarité. "La vocation foncière de l'Etat social ne se résume pas à la simple répartition de moyens financiers ", expliquait le chancelier en mars 2004.

Bien qu'une large partie de l'opinion ait approuvé cette réforme - la seule réellement structurelle des gouvernements Schröder I et II -, une grande partie des militants et de l'électorat du SPD s'est sentie trahie par le cap du chancelier, décidément trop 'libéral' à son goût. Gerhard Schröder a perdu non seulement le soutien de la base de son parti, mais aussi du mouvement syndical. A l'approche des élections en Rhénanie du Nord-Westphalie de mai 2005, Franz Müntefering a certes été chargé de rallier les militants 'de gauche' du SPD en lançant opportunément un débat sur le " capitalisme » et en montrant du doigt ces hedge fonds qui tombent sur les entreprises allemandes comme des " essaims de sauterelles ". Mais le SPD n'en perd pas moins les élections en Rhénanie du Nord-Westphalie - son fief, et le dernier Land encore gouverné par une coalition SPD/Verts. Gerhard Schröder en tirera les conclusions et demandera la dissolution du Bundesrat, provoquant des élections anticipées pour reconquérir une confiance plus large à la fois auprès de son parti et au-delà.

IL AURA FALLU DEUX MANDATS au gouvernement de coalition SPD/Verts pour définir une politique réaliste. II avait placé le premier sous le signe d'une "nouvelle politique économique ", voulant faire de l'Allemagne le laboratoire d'un maximalisme écologiste doublé de néo-keynesianisme. Mais très vite, il a dû se rendre à l'évidence que le jeu institutionnel national comme l'intégration croissante de l'UE exigeaient une nouvelle approche pour relever les défis de la globalisation ou du vieillissement démographique. Et c'est finalement au nom de la compétitivité globale qu'il osera affronter le changement, lançant enfin les réformes structurelles que réclamaient depuis longtemps les experts mais que la gouvernement Kohl, trop préoccupé par le chantier de l'unification, ne pouvait pas encore amorcer. A l'innovation du premier mandat succède après 2002 le début d'une refonte du contrat social allemand. Entre-temps, la coalition a assumé sa responsabilité dans le concert européen.

Mais cette approche 'néo-réaliste' n'a jamais été acquise. Les nombreuses contradictions des orientations proposées attestent de la difficulté à définir une politique cohérente au sein d'une majorité 'plurielle'. Les revers électoraux successifs de la coalition SPD/Verts dans les Länder, l'échec du SPD dans son fief de Rhénanie du Nord-Westpahlie et son maigre résultat aux parlementaires fédérales en septembre 2005 montrent combien il est difficile de mener une politique de réformes au long cours malgré un calendrier électoral chargé. II n'en reste pas moins que, sous l'impulsion de Gerhard Schröder, la coalition $\mathrm{SPD} /$ Verts aura réussi à amorcer le changement. L'Allemagne s'est réapproprié les valeurs fondatrices d'un "modèle rhénan » qui fut source de prospérité et de solidarité, acceptant un douloureux processus de réformes au nom de la compétitivité de son économie comme de l'équité de son modèle social.

\section{Indications bibliographiques:}

Bourgeols l., "Inerties et volonté de réformes ", in DemesmaY C., STARK H. (eds.), Qui dirige l'Allemagne ? Presses universitaires du Septentrion, 2005

Commissariat General du PLAN / DFI, Compétitivité globale: une perspective francoallemande, Rapport du Groupe franco-allemand dur la compétitivité, Paris, 2001

Commun P. (dir), L'ordolibéralisme allemand. Aux sources de l'économie sociale de marché, CIRAC, 2003

LESTRADE B., "La politique gouvernementale pour l'emploi, de Schröder I à Schröder II ", in BOURGEOIS I. (dir), Le modèle social allemand en mutation, CIRAC, 2005

«Regierungsbilanz. 7 Jahre Rot-Grün», Frankfurter Allgemeine Zeitung, dossier (www.faz.de)

SACHVERSTÄNDIGENRAT ZUR BEGUTACHTUNG DER GESAMTWIRTSCHAFTLICHEN ENTWICKLUNG, rapports annuels (www.sachverstaendigenrat.org)

SCHRCEDER W., "Le modèle syndical allemand n'existe plus ", in BouRGEOIs I. (dir), Le modèle social allemand en mutation, CIRAC, 2005

www.bundesregierung.de 